\title{
Image Restoration Based on Stochastic Resonance in a Parallel Array of Fitzhugh-Nagumo Neuron
}

\author{
Huage Zhang $\mathbb{D},{ }^{1}$ Jinfei Yu, ${ }^{2}$ Yumei Ma $\mathbb{D}^{1},{ }^{1}$ Zhenkuan Pan, ${ }^{1}$ and Jingjing Zhao $\mathbb{D}^{1}$ \\ ${ }^{1}$ College of Computer Science \& Technology, Qingdao University, Qingdao 266071, Shandong, China \\ ${ }^{2}$ Zibo Technician College, Zibo 255000, Shandong, China \\ Correspondence should be addressed to Yumei Ma; mayumei_qdu@163.com
}

Received 11 August 2020; Revised 22 September 2020; Accepted 24 October 2020; Published 12 November 2020

Academic Editor: Jing $\mathrm{Na}$

Copyright ( 2020 Huage Zhang et al. This is an open access article distributed under the Creative Commons Attribution License, which permits unrestricted use, distribution, and reproduction in any medium, provided the original work is properly cited.

The poor denoising effect for noisy grayscale images with traditional processing methods would be obtained under strong noise condition, and some image details would be lost. In this paper, a parallel array model of Fitzhugh-Nagumo (FHN) neurons was proposed, which can restore noisy grayscale images well with low peak signal-to-noise ratio (PSNR) conditions and the image details are better preserved. Firstly, the row-column scanning method was used to convert the 2D grayscale image into a 1D signal, and then the 1D signal was converted into a binary pulse amplitude modulation (BPAM) signal by signal modulation. The modulated signal was input to an FHN parallel array for stochastic resonance (SR). Finally, the array output signal was restored to a 2D gray image, and the image restoration effect was analyzed based on the PSNR and Structural SIMilarity (SSIM) index. It is shown that the SR effect can be exhibited in an array of FHN neuron nonlinearities by increasing the array size, and the image restoration effect is significantly better than the traditional image restoration method, and larger PSNR and SSIM can be obtained. It provides a new idea for grayscale image restoration in a low PSNR environment.

\section{Introduction}

The image can be affected by noise in the process of acquisition and transmission, resulting in a decline in image quality. At present, traditional image restoration methods [1-3] such as filtering mainly focus on suppressing and reducing noise, but some image information will be lost while denoising. With the development of nonlinear dynamics, SR phenomena are well demonstrated wherein the response of nonlinear systems can be enhanced with the presence of internal or external noise.

The concept of SR was first proposed by Benzi to explain the periodic changes in the glacial period and warm climate period in ancient meteorology $[4,5]$. Thus, the research of nonlinear systems has been rapidly developed [6, 7]. On the one hand, SR has been widely applied in the field of imaging processing $[8,9]$. The adaptive adjustment of bistable system parameters is used for image restoration $[10,11]$ and the MR image can be enhanced by the method of SR neuron model [12]. Then, aperiodic stochastic resonance is used to restore the grayscale image in [13], and underwater scattering images can be reconstructed by the SR method in [14]. However, the desired effect cannot be achieved with these methods under low PSNR environment. On the other hand, nonlinear systems are widely used in control engineering $[15,16]$, laying the foundation for the development of SR in nonlinear systems. The remarkable results have been achieved by the parallel array of nonlinearities in the field of SR. In 1995, the theory of array SR was proposed by Lindner et al. [17] and the results showed that the output signal-tonoise ratio can be improved with array SR. The parallel bistable system was found by Wang et al. [18] to detect interference characteristic signals at a lower input signal-tonoise ratio. In the cascaded bistable system, perturbation characteristic signals with lower input signal-to-noise ratio can be detected and a parallel array model based on threshold and a saturated parallel array model are proposed [19]. Wang et al. [20] used array stochastic resonance to improve the reliability and robustness of logical stochastic resonance under colored noise. 
SR is also widely used in the fields of chemistry [21], biology [22], and physics [23, 24]. In the field of neuroscience [25], a single neuron model is the basis for studying the biological and electrical properties of neurons. The simplified 2D Fitzhugh-Nagumo model was proposed by Fitzhugh and Nagumo based on the 4D Hodgkin-Huxley (HH) model $[26,27]$. Then, the $1 \mathrm{D}$ FHN model was proposed by Alarcon et al. [28] by simplifying the 2D FHN model. SR in the nervous system is being studied by more and more researchers and SR has become a hot research topic in biological neural signal processing. In 1991, Longtin used theoretical models to simulate and study the phenomenon of integer multiple discharge rhythms and concluded that the rhythm is related to SR effects. The concept of nonperiodic SR was proposed by Collins to describe the phenomenon of SR in FHN when studying the neural model of biostimulation [29]. The generation and regulation mechanism of SR in the nervous system, which depends on the frequency difference, is revealed by Guo et al. [30]. The SR effect in FHN neurons was detected by Yao et al. [31] and stochastic multiple resonance was found in [32] in the study of coupled excitation of FHN neurons, which can effectively detect subthreshold signals. A command filter adaptive neural network control method for multi-input multioutput nonlinear systems was proposed by $\mathrm{Yu}$ et al. [33].

In this paper, a parallel array model of FHN neuron based on SR is proposed for image restoration. A 2D image signal is converted into a $1 \mathrm{D}$ signal through row and column scanning, and then the $1 \mathrm{D}$ signal is transformed into a $1 \mathrm{D}$ binary pulse amplitude modulation aperiodic signal through pulse amplitude modulation. Then the 1D BPAM aperiodic signal is input to the FHN array nonlinearities, and finally, the output signal is decoded, demodulated, and restored to obtain the restored image. The main works of this paper are as follows: (1) The FHN neuron and the array SR are combined to establish an array SR image restoration model based on the FHN neuron and make full use of the changes in the dynamic characteristics of the neuron caused by noise, enhance the sensitivity of the neuron, and improve the stochastic resonance of the model performance. (2) The FHN neuron parallel array method is compared with the traditional filtering and 2D SR methods in multi-image images. The results show that the method effectively restores the visual effect and PSNR performance of noisy images in a low PSNR environment.

\section{System Model and Performance Evaluation}

2.1. System Model Design. The model in this section is a grayscale image restoration model based on array FHN neurons, and the experimental flowchart is shown in Figure 1. In image processing, the general image degradation model caused by noise is described as follows:

$$
f(i, j)=s(i, j)+\xi(i, j) \text { for } 1 \leq i \leq M, 1 \leq j \leq N,
$$

where $s(i, j)$ is the original $M \times N$ grayscale image and $\xi(i, j)$ is Gaussian white noise with zero mean and variance of $\sigma_{\xi}^{2} \cdot f(i, j)$ is a degraded image polluted by noise. In order to improve the performance of degraded images, it can be applied to FHN nonlinear parallel array, the process steps are as follows.

2.1.1. Original Image Processing. Firstly, the image is subjected to dimensionality reduction by row or column directional scanning, so that the original grayscale image is converted into a $1 \mathrm{D}$ signal of length $H_{1 \times M N}(M$ and $N$ are the rows and columns of the original grayscale image). Then the $1 \mathrm{D}$ image sequence $H_{1 \times M N}$ is encoded as an eight-bit binary sequence $Q_{1 \times 8 M N}$ consisting of 0 and 1 with a length of $8 \times M \times N$.

2.1.2. Modulation. Next, the binary sequence $Q_{1 \times 8 M N}$ is subjected to signal modulation processing into a $1 \mathrm{D}$ bipolar aperiodic signal $s(i, j)$ by the following equation:

$$
s(t)=A \sum_{l=1}^{8 M N} W_{l} G\left(t-k T_{b}\right)
$$

where $A$ denotes the amplitude of the $\operatorname{signal} s(i, j)$ and $G$ is a rectangular pulse with a period of $T_{b}$. When $t \in\left(0, T_{b}\right)$, $G(t)=1$, and otherwise, $G(t)=0 . W_{l}(l=1,2, \cdots \infty, 8 M N)$ is a $1 \mathrm{D}$ sequence of values -1 and 1 obtained by performing polarity conversion on binary symbols $Q_{1 \times 8 M N}(0 \rightarrow-1,1 \rightarrow 1) . k$ represents the size of the image, that is, the size of the image is $2^{\mathrm{k}} \times 2^{\mathrm{k}}$.

2.1.3. Array SR Processing. The $2 \mathrm{D}$ FHN neuron model is as follows:

$$
\left\{\begin{array}{l}
\frac{\mathrm{d} v}{\mathrm{~d} t}=v(a-v)(v-1)-w+I_{a} \\
\frac{\mathrm{d} w}{\mathrm{~d} t}=b v-\gamma w
\end{array}\right.
$$

where $v$ represents the fast-changing membrane voltage variable, $0<a<1$ is the threshold, $b$ and $\gamma$ are normal numbers. Here is the slow-changing return variable $w, I_{a}$ is the excitation current, and when $I_{a}=0$, a simplified $1 \mathrm{D}$ FHN neuron model can be obtained [34] as follows:

$$
\frac{\mathrm{d} v}{\mathrm{~d} t}=v(a-v)(v-1)-\frac{b}{\gamma} v+\xi(t),
$$

where $\xi(t)$ is Gaussian white noise. The $1 \mathrm{D}$ FHN neuron model is used as the array unit of the parallel array and has the following equation:

$$
\frac{\mathrm{d} v_{i}(t)}{\mathrm{d} t}=v_{i}(t)\left(a-v_{i}(t)\right)\left(v_{i}(t)-1\right)-\frac{b}{\gamma} v_{i}(t)+h(t) .
$$

where $i=1,2, \cdots, L$, and parameter $L$ is the size of parallel FHN neurons. Each SR array unit is an FHN neuron and input $h(t)=s(t)+\xi_{n}(t)(n=1,2,3, \cdots L)$ to the FHN array unit. Then $x_{n}(t)$ can be obtained after processing by the FHN nonlinearity unit, and $x_{n}(t)$ is arithmetically averaged 


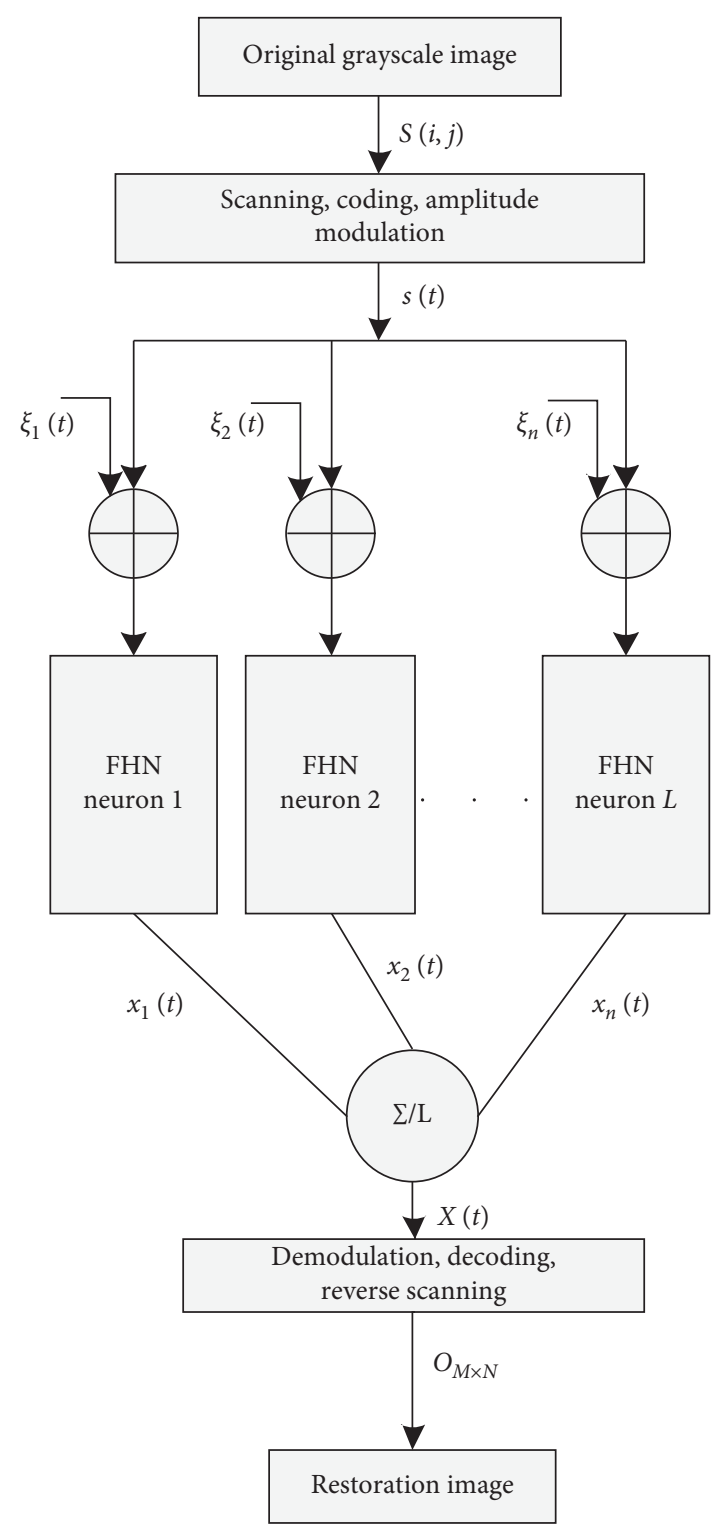

FIgURE 1: The flowchart of the FHN neuron array model.

and processed. Finally, the output of the FHN parallel array is $X(t)$ :

$$
X(t)=\frac{1}{L} \sum_{n=1}^{L} x_{n}(t)
$$

2.1.4. Demodulation. Each new signal $W_{l}$ starts at time $t_{k}=$ $k T_{b}$ with a duration of $T_{b}$. So, the time interval of the $1 \mathrm{D}$ aperiodic signal $s(t)$ is $T_{b}$. The optimal demodulation scheme is as follows:

$$
Y_{k}=\left\{\begin{array}{l}
\operatorname{sign}\left(X\left(t_{k}+T_{b}\right)\right)=1, X\left(t_{k}+T_{b}\right) \geq 0, \\
\operatorname{sign}\left(X\left(t_{k}+T_{b}\right)\right)=0, X\left(t_{k}+T_{b}\right)<0 .
\end{array}\right.
$$

2.1.5. Decoding and Restoring Images. The demodulated binary 1D signal $Y_{1 \times 8 M N}$ is decoded and then $Y_{1 \times 8 M N}$ is inversely scanned by column or row to obtain a restored image $O_{M \times N}$.

The above is the FHN array model and the processing of image restoring. The next part is the performance evaluation.

2.2. Image Performance Evaluation. In most image processing, PSNR is described as an objective criterion for evaluating image quality. It is defined as follows:

$$
\text { PSNR }=10 \times \log _{10} \frac{255^{2}}{\mathrm{MSE}^{2}},
$$

where MSE is the mean square error between the original image and the processed image, which is defined as follows:

$$
\operatorname{MSE}=\frac{1}{M N} \sum_{i=1}^{M} \sum_{j=1}^{N}[o(i, j)-s(i, j)]^{2},
$$

where $M$ and $N$ represent the rows and columns of the $2 \mathrm{D}$ matrix of the gray image, $s(i, j)$ is the gray value of the original image pixel, and $o(i, j)$ is the gray value of the restored image pixel. Generally, a large PSNR value presents a better recovery effect.

\section{Experimental Results and Analysis}

In order to demonstrate the restoration effect of the parallel array of FHN neurons on the image, Wiener filtering, mean filtering, and 2D stochastic resonance [35] method are selected for experimental comparison and the noise used in this paper is Gaussian white noise. The $2 \mathrm{D}$ stochastic resonance method is that the original noisy image is scanned by the row or column to obtain a $1 \mathrm{D}$ signal and then input into a single FHN neuron, and the output 1D signal is restored to a $2 \mathrm{D}$ image. Then, the restored image is processed by the above treatment again, and the final recovered image can be obtained. The Wiener2 function in MATLAB is used for Wiener filtering and the $3 \times 3$ size filter template is used for average filtering. Then the three noisy grayscale images of different sizes $(128 \times 128,256 \times 256$, and $512 \times 512)$ are processed by these comparison methods and parallel array of FHN neurons.

In actual simulation, we consulted references and perform a large number of experiments to determine $\gamma=10^{-4}$, $a=0.5$, and $b=1$ as experimental parameters of FHN neurons. In the same picture, the size of the array is $L$. In the image processing process in this paper, the FHN parallel array fixes other parameters to adjust the size of the array $L$. It can be seen that the image restoration effect varies with the size of the array $L$.

\subsection{Experimental Data}

3.1.1. House Image. The noise with zero mean and a variance of 0.6 is added to the house image $(128 \times 128)$. The PSNR of a noisy house image is $7.6193 \mathrm{~dB}$. 


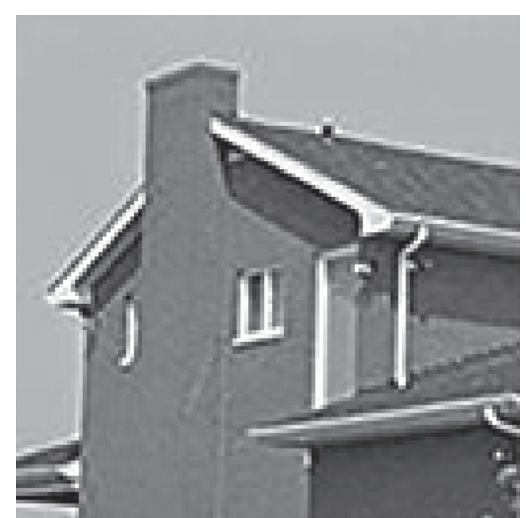

(a)

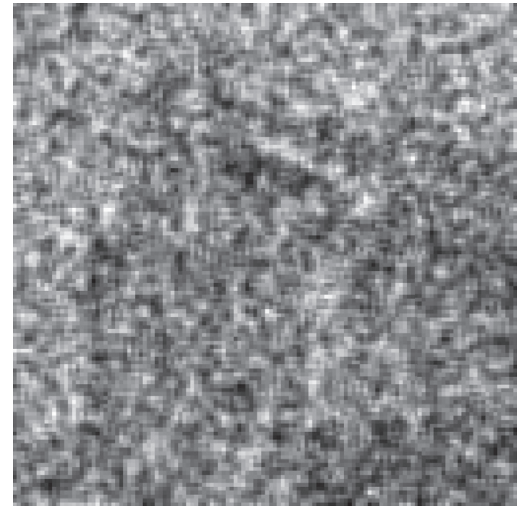

(d)

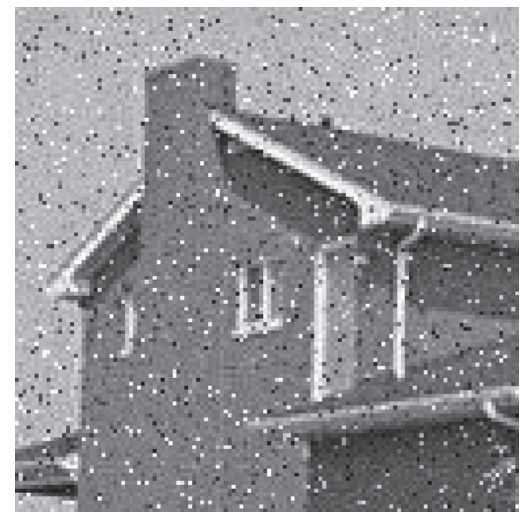

(g)

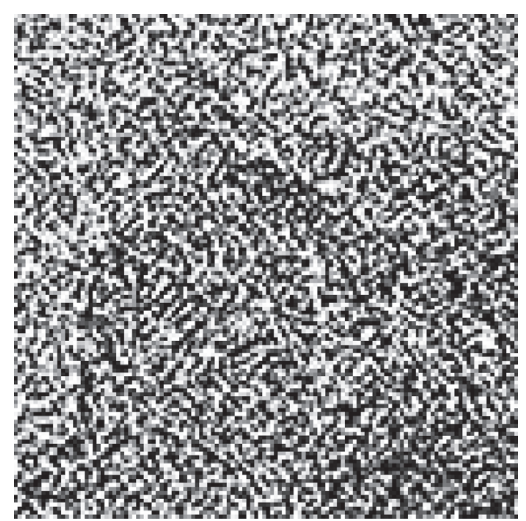

(b)

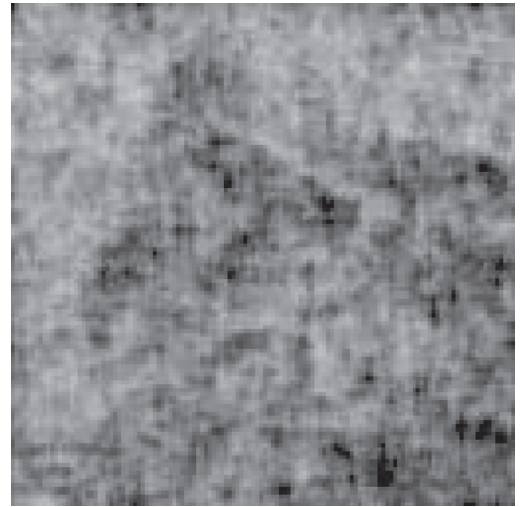

(e)

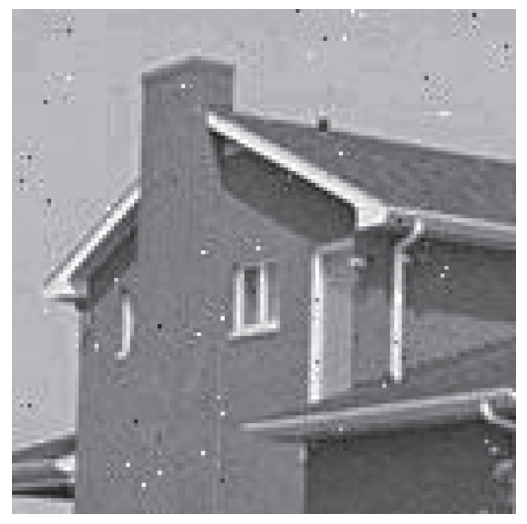

(h)

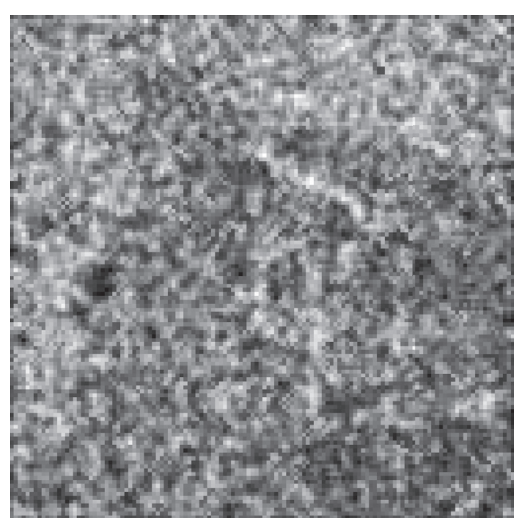

(c)

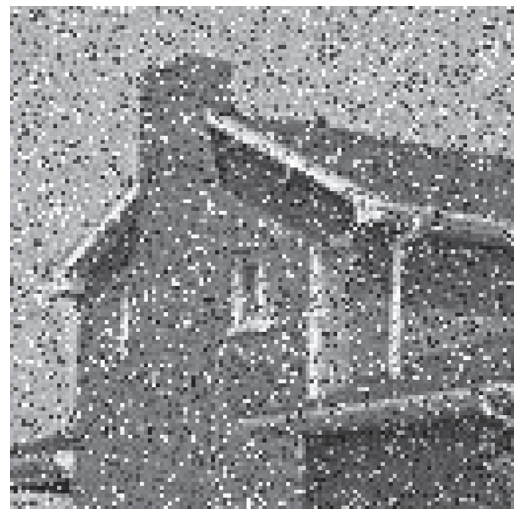

(f)

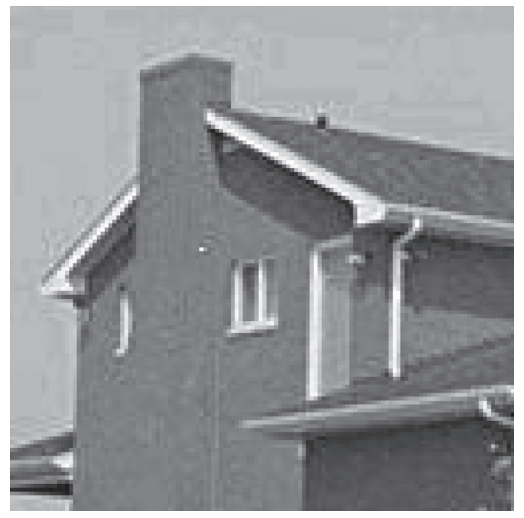

(i)

FIGURE 2: House images with different image restoration methods: (a) original house image, (b) noisy house image, (c) Wiener filtering, (d) mean filtering, (e) 2D stochastic resonance, (f) array size: $L=1,(\mathrm{~g})$ array size: $L=2$, (h) array size: $L=5$, and (i) array size: $L=10$.

Figure 2 shows the comparison of restored images among the two classical filtering methods, 2D SR method, and FHN neuron parallel array method. Figures $2(\mathrm{c})-2(\mathrm{e})$ show the restoration effect by two classical filtering methods and 2D SR method. Obviously, the image restoration effect is not very effective and the noisy house image with low PSNR cannot be restored well. Figures 2(f)-2(i) show the restoration effect of the parallel array of FHN neurons on the noisy house image. It shows that as the array size increases, the SR effect and image restoration effect are better. The results show that the larger the array size, the SR effect and the image restoration effect can be gradually improved. As shown in Figure 2(i), when $L=10$, the restored house image is almost the same as the original house image.

Table 1 shows the PSNR performance comparison of restored house images under different restoration methods. Obviously, the PSNR of the classic filtering methods and 2D SR methods are similar and the recovery effect is not very 
TABLE 1: PSNR value of noisy house image under different restoration methods.

PSNR (dB)

Image name Image size

Noisy house Wiener filtering Mean filtering 2D stochastic resonance

The proposed method

\begin{tabular}{|c|c|c|c|c|c|c|c|c|c|}
\hline & & & & & & $L=1$ & $L=2$ & $L=5$ & $L=10$ \\
\hline House & $128 \times 128$ & 7.6193 & 15.2623 & 14.4978 & 16.2076 & 15.2736 & 19.5306 & 30.3087 & 35.7975 \\
\hline
\end{tabular}

effective. When the parallel array of FHN neuron size is $L=1$, the PSNR value of the parallel array of the FHN neuron SR method is similar to the classic filtering and 2D stochastic resonance methods. As the size of the FHN array increases, the visual effect and performance index of the restored image also increase. When the array size $L=10$, the visual effect of the restored house image is excellent, and $\mathrm{PSNR}=35.7975 \mathrm{~dB}$.

3.1.2. Rice Image. The noise with zero mean and a variance of 0.4 is added to the rice image $(256 \times 256)$. The PSNR of a noisy rice image is $8.2193 \mathrm{~dB}$.

Figure 3 shows the comparison of restored images between two classical filtering methods, 2D SR method, and FHN neuron parallel array method. The results show that as the size of the array increases, the SR effect and image restoration effect are better. Compared with the two classical filtering and 2D SR methods, the image restoration effect has been significantly improved.

Table 2 shows the PSNR performance comparison of restored rice images under different restoration methods. When the parallel array of FHN neuron size is $L=1$, the PSNR value of the parallel array of the FHN neuron SR method is similar to the classic filtering and 2D SR methods, and as the array size becomes larger, the gap between them becomes more and more obvious. It shows that this is consistent with the nonlinear nature of SR, which converts noise signals into energy signals.

3.1.3. Einstein Image. The noise with zero mean and a variance of 0.8 is added to the Einstein image $(512 \times 512)$. The PSNR of a noisy Einstein image is $7.4014 \mathrm{~dB}$.

Figure 4 shows the comparison of restored images between two classical filtering methods, 2D SR method, and FHN neuron parallel array method. Compared with the two classic filtering and 2D stochastic resonance methods, the image restoration effect has been significantly improved. However, in the case of parallel arrays of FHN neurons of the same size, the restoration effect of the Einstein image is not as good as that of the house image and the rice image. It shows that as the pixel size of the image and the noise intensity increase, the restoration effect of the noise image will also be affected. As the array becomes larger, the visual effect of the restored image is comparable to the original image. But the computational complexity and the time required to process the image will also increase. Therefore, we need to weigh the computational complexity and the demand for image restoration effects to select the most suitable size of the FHN parallel array.
Table 3 shows the PSNR performance comparison of restored Einstein images under different restoration methods. It can be seen that the PSNR values of the classic filtering and 2D SR methods are similar, and the restoration effect is not effective. The parallel array of FHN neuron parallel array methods has a lower PSNR value than the classic filtering and 2D SR methods when the array size $L=1$. When the array size $L=10, \mathrm{PSNR}=34.9538 \mathrm{~dB}$, and the PSNR value of the restored Einstein image is significantly higher than that of the other three methods.

3.2. Bit Error Rate Analysis. In order to further analyze the recovery effect of the parallel array of FHN neurons, we analyzed the bit error rate (BER) of the model output. BER is often used as an indicator of data communication quality. The calculation formula is as follows:

$$
\mathrm{BER}=\frac{\mathrm{RN}}{\mathrm{TN}}
$$

where $\mathrm{RN}$ is the number of error bits and TN is the total number of bits.

Figure 5 shows the BER curve of the house image $(128 \times 128)$ as a function of the noise intensity with different array sizes of the FHN array. In Figure 5, it can be seen that as the noise intensity increases, the BER also increases. But the size of the FHN array has a significant effect on the BER. The smaller the parallel array of FHN neurons, the higher the BER. As the array size of FHN neurons array increases, the BER decreases significantly. Therefore, the bit error rate can be reduced by increasing the array size of the FHN neurons array and the accuracy of the output signal can be enhanced.

3.3. Structural SIMilarity Analysis. SSIM is an index to measure the similarity of two images. The calculation formula is as follows:

$$
\operatorname{SSIM}(x, y)=\frac{\left(2 \mu_{x} \mu_{y}+c_{1}\right)\left(2 \sigma_{x y}+c_{2}\right)}{\left(\mu_{x}^{2}+\mu_{y}^{2}+c_{1}\right)\left(\sigma_{x}^{2}+\sigma_{y}^{2}+c_{2}\right)},
$$

where $x$ and $y$ are the original grayscale image and the restore image, $\mu_{x}$ is the average of $x, \mu_{y}$ is the average of $y, \sigma_{x}$ is the variance of $x$, and $\sigma_{y}$ is the variance of $y . \sigma_{x y}$ represents the covariance of $x$ and $y$. Constant $c_{1}=\left(k_{1} L\right)^{2}$ and $c 2=\left(k_{2} L\right)^{2} . L$ is the dynamic range of the pixel value. $k_{1}=$ 0.01 and $k_{2}=0.03$. House images, rice images, and Einstein images were analyzed by SSIM, and the results are shown in Table 4.

It can be seen from Table 4 that the SSIM value increases with the increase of the array size. When the array size 


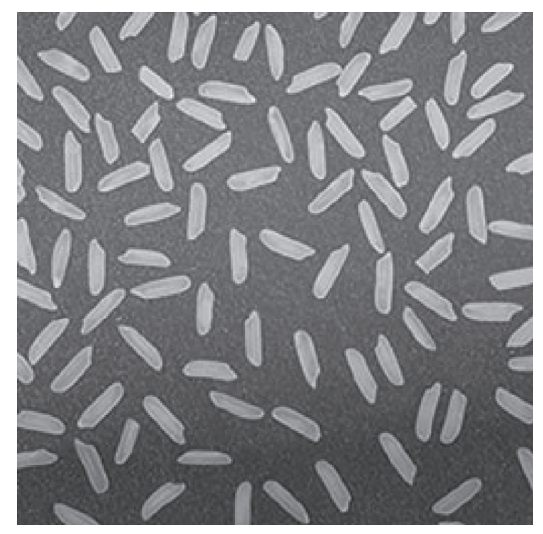

(a)

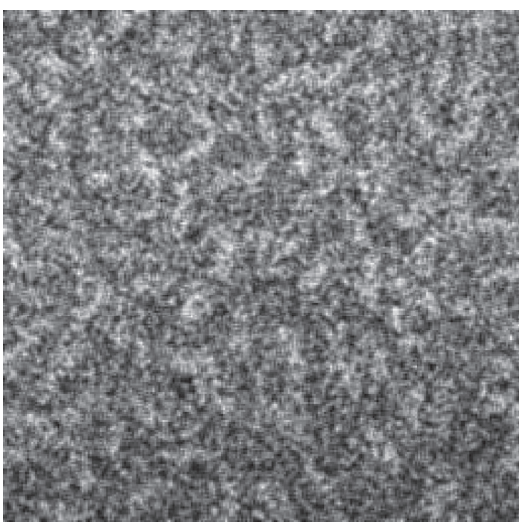

(d)

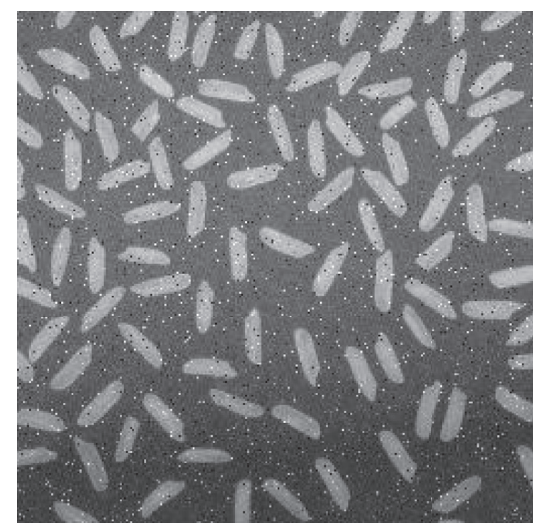

(g)

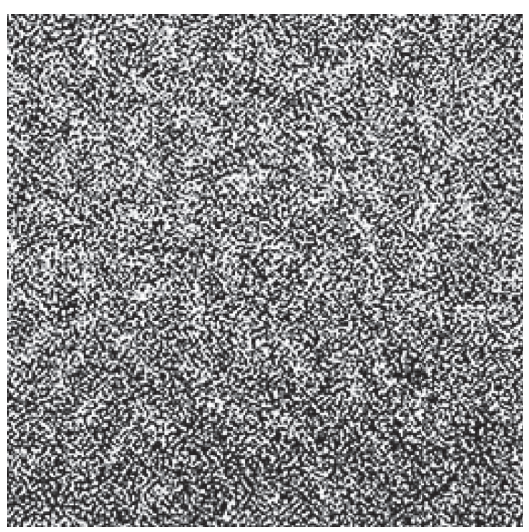

(b)

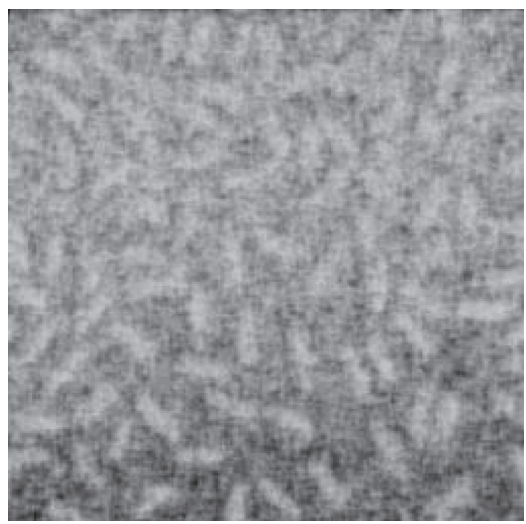

(e)

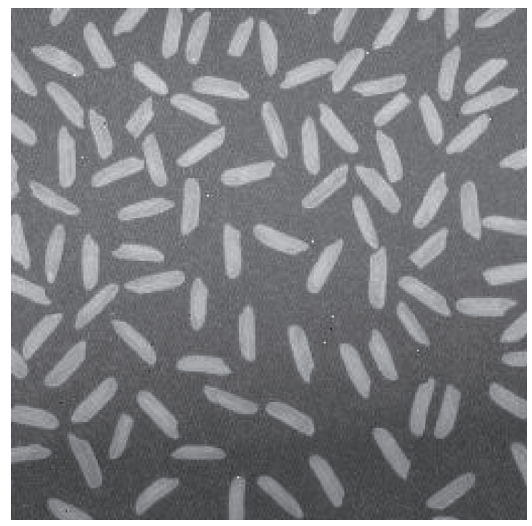

(h)

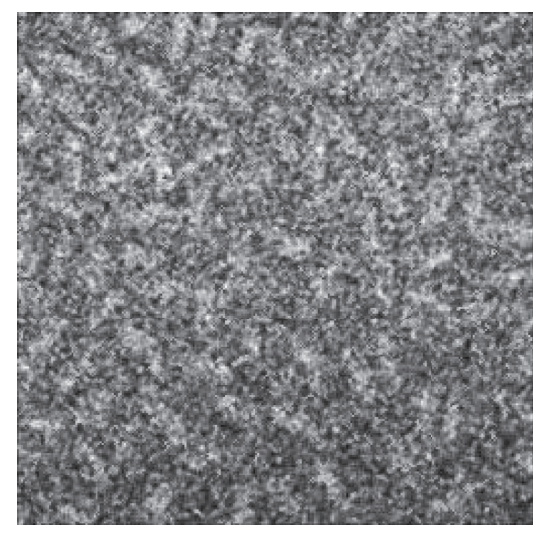

(c)

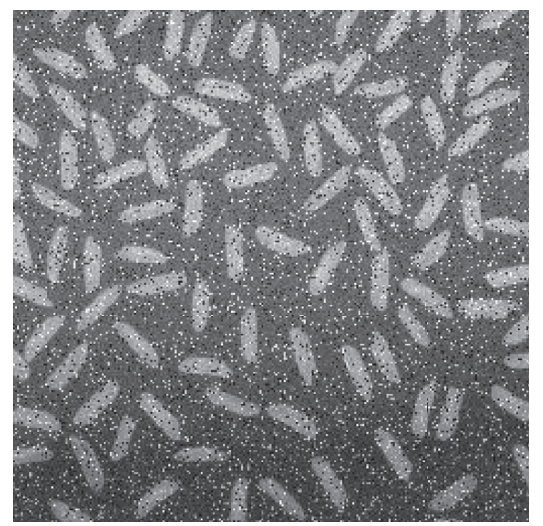

(f)

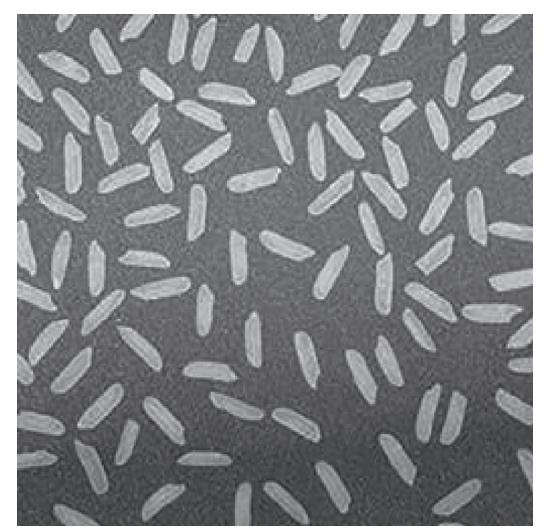

(i)

FIGURE 3: Rice images with different image restoration methods: (a) original rice image, (b) noisy rice image, (c) Wiener filtering, (d) mean filtering, (e) 2D stochastic resonance, (f) array size: $L=1,(\mathrm{~g})$ array size: $L=2$, (h) array size: $L=5$, and (i) array size: $L=10$.

TABLE 2: PSNR value of noisy rice image under different restoration methods.

PSNR $(\mathrm{dB})$

Image name Image size

Noisy rice Wiener filtering Mean filtering 2D stochastic resonance
The proposed method

$L=1 \quad L=2 \quad L=5 \quad L=10$

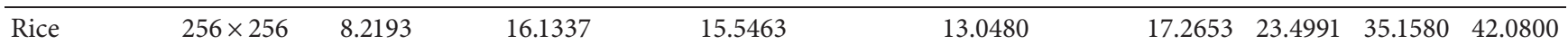




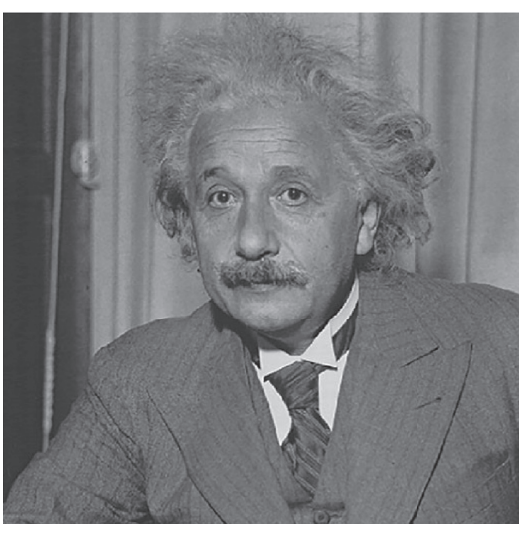

(a)

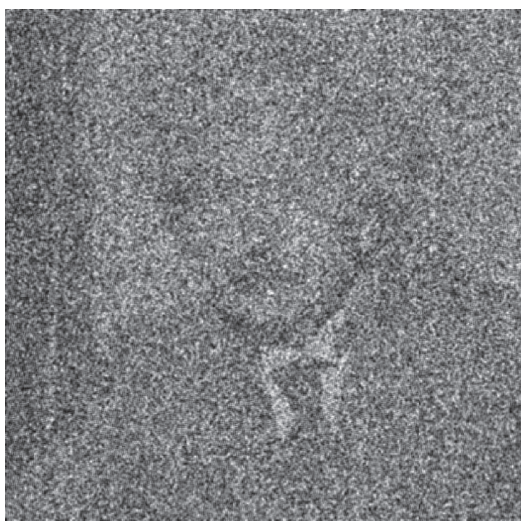

(d)

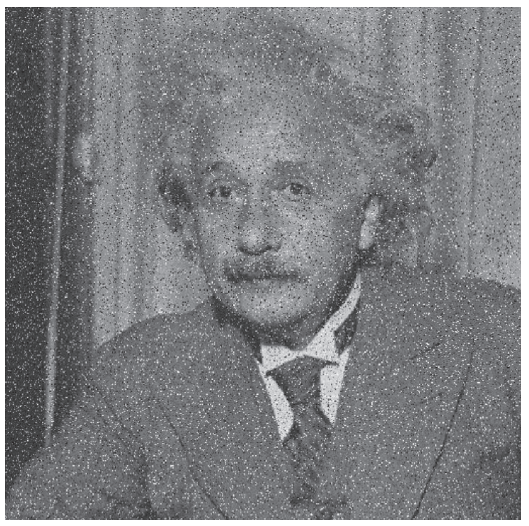

(g)

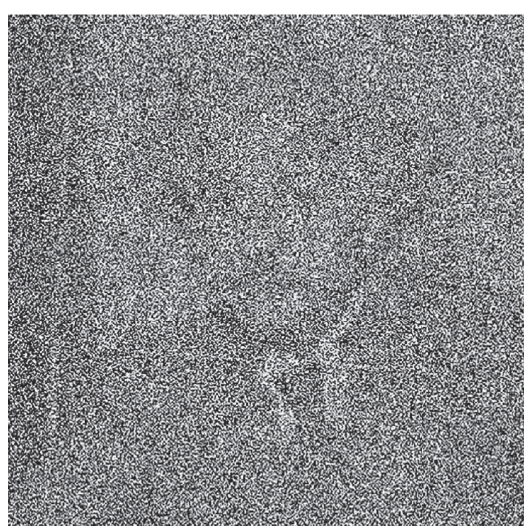

(b)

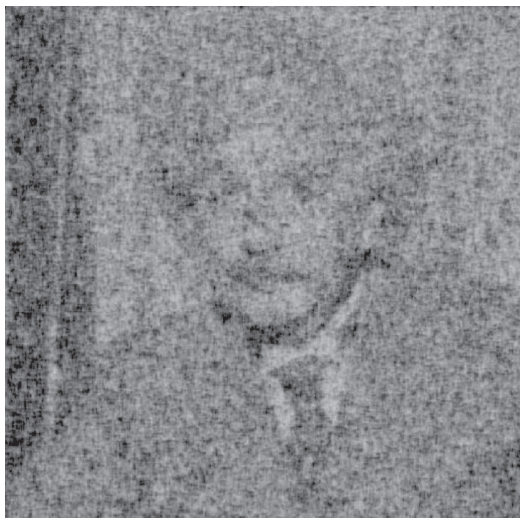

(e)

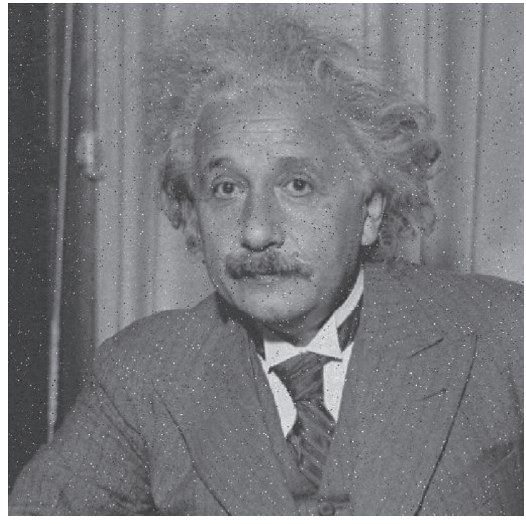

(h)

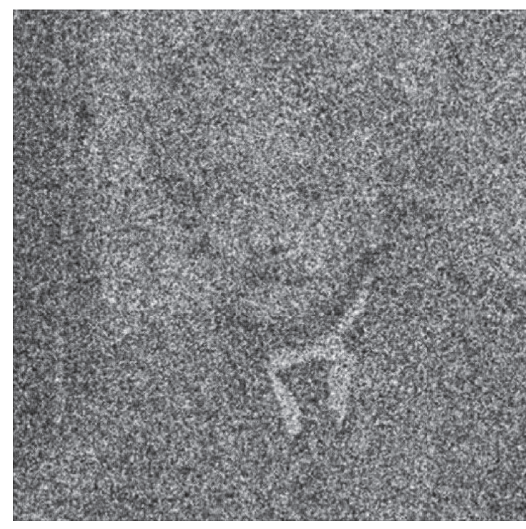

(c)

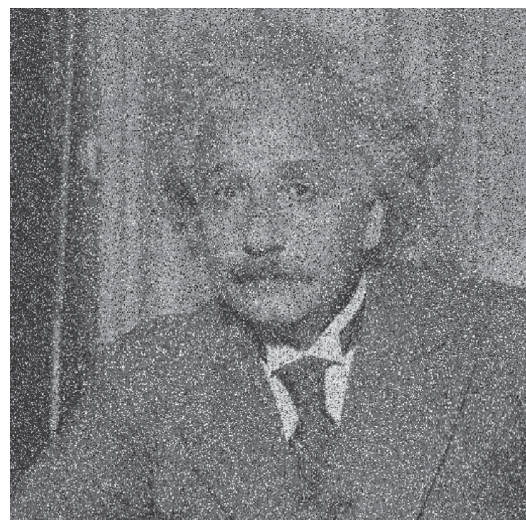

(f)

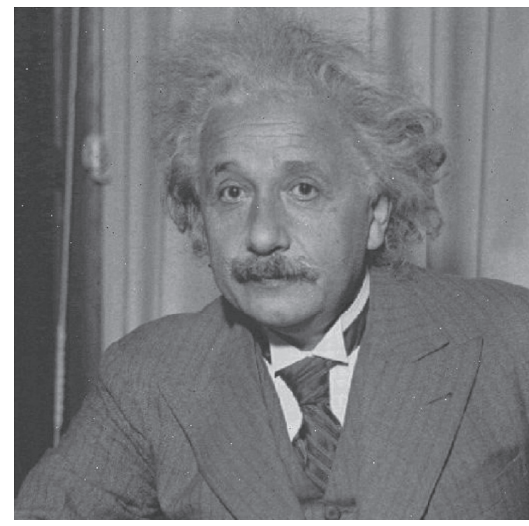

(i)

FIGURE 4: Einstein images with different image restoration methods: (a) original Einstein image, (b) noisy Einstein image, (c) Wiener filtering, (d) mean filtering, (e) 2D stochastic resonance, (f) array size: $L=1,(\mathrm{~g})$ array size: $L=2$, (h) array size: $L=5$, and (i) array size: $L=10$.

TABLE 3: PSNR value of noisy Einstein image under different restoration methods.

\begin{tabular}{|c|c|c|c|c|c|c|c|c|c|}
\hline \multirow{3}{*}{ Image name } & \multirow{3}{*}{ Image size } & \multicolumn{8}{|c|}{ PSNR (dB) } \\
\hline & & \multirow{2}{*}{ Noisy Einstein } & \multirow{2}{*}{$\begin{array}{l}\text { Wiener } \\
\text { filtering }\end{array}$} & \multirow{2}{*}{ Mean filtering } & \multirow{2}{*}{ 2D stochastic resonance } & \multicolumn{4}{|c|}{ The proposed method } \\
\hline & & & & & & $L=1$ & $L=2$ & $L=5$ & $L=10$ \\
\hline Einstein & $512 \times 512$ & 7.4014 & 15.1588 & 15.6554 & 16.8012 & 13.9864 & 17.2597 & 26.3254 & 34.9538 \\
\hline
\end{tabular}




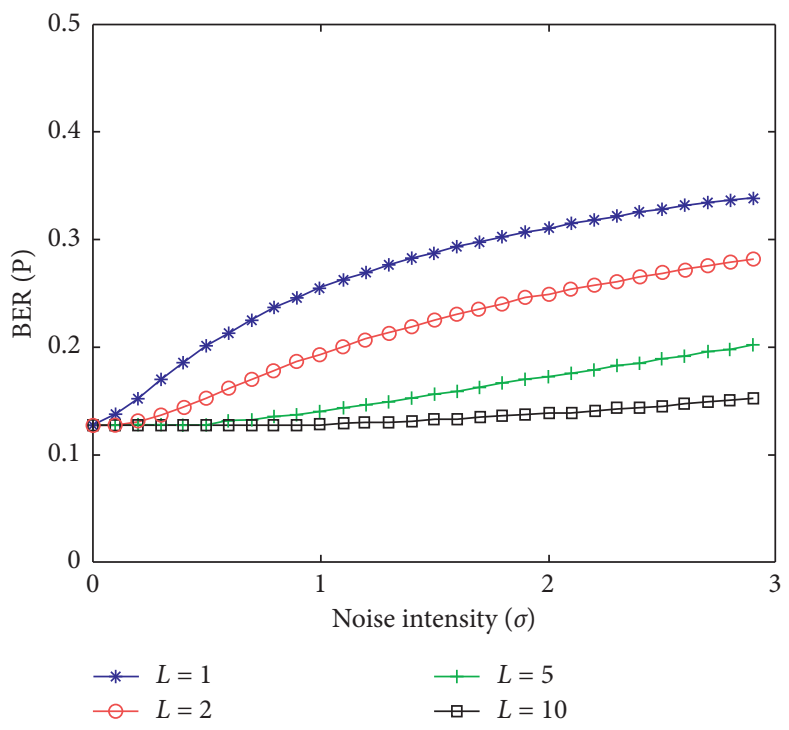

FIGURE 5: BER of the parallel array of FHN neurons with different array size $(L=1,2,5$, and 10$)$.

TABLE 4: SSIM values of different restoration methods.

\begin{tabular}{lccc}
\hline Recovery method & SSIM & \\
& House & Rice & Einstein \\
\hline Noisy image & 0.0428 & 0.0481 & 0.0584 \\
Mean filtering & 0.1559 & 0.2178 & 0.1359 \\
Wiener filtering & 0.1482 & 0.1938 & 0.1281 \\
2D SR & 0.2142 & 0.2907 & 0.2535 \\
FHN array $L=1$ & 0.2219 & 0.3210 & 0.2407 \\
FHN array $L=2$ & 0.3895 & 0.6215 & 0.4058 \\
FHN array $L=5$ & 0.8351 & 0.8723 & 0.8155 \\
FHN array $L=10$ & 0.9223 & 0.9679 & 0.9574 \\
\hline
\end{tabular}

$L=10$, SSIM can reach more than 0.92 , and the image restoration effect is excellent.

\section{Conclusion}

In this paper, the method of FHN neuron parallel array is proposed to improve the transmission quality of low PSNR noisy images. Experimental results show that compared with traditional filtering and 2D SR methods, the image quality and PSNR value can be enhanced excellently. With the increase of the parallel array size of FHN neurons, the enhancement effect is more excellent. In this paper, this method is adopted to process three kinds of noisy images with different pixel sizes, and the visual effect and PSNR value of the restored image are improved greatly. This method has potential applications in remote sensing detection, transmission systems, target detection, etc.

\section{Data Availability}

The data used to support the findings of this study are available from the corresponding author upon request.

\section{Conflicts of Interest}

The authors declare that there are no conflicts of interest regarding the publication of this paper.

\section{Acknowledgments}

This work was partially supported by the National Natural Science Foundation of China (61501276, 61573204, 61772294, and 61973179), the China Postdoctoral Science Foundation (2016M592139), and the Qingdao Postdoctoral Applied Research Project (2015120).

\section{References}

[1] Z. Huang, Y. Zhang, Q. Li, T. Zhang, N. Sang, and H. Hong, "Progressive dual-domain filter for enhancing and denoising optical remote-sensing images," IEEE Geoscience and Remote Sensing Letters, vol. 15, no. 5, pp. 759-763, 2018.

[2] U. Erkan, D. N. H. Thanh, L. M. Hieu, and S. Enginoglu, "An iterative mean filter for image denoising," IEEE Access, vol. 7, pp. 167847-167859, 2019.

[3] Y. Jin, W. Jiang, J. Shao, and J. Lu, "An improved image denoising model based on nonlocal means filter," Mathematical Problems in Engineering, vol. 2018, Article ID 8593934, 8 pages, 2018.

[4] R. Benzi, A. Sutera, and A. Vulpiani, "The mechanism of stochastic resonance," Journal of Physics A: Mathematical and General, vol. 14, no. 11, pp. L453-L457, 1981.

[5] R. Benzi, G. Parisi, A. Sutera, and A. Vulpiani, "Stochastic resonance in climatic change," Tellus, vol. 34, no. 1, pp. 10-15, 1982.

[6] J. Yu, L. Zhao, H. Yu, and C. Lin, "Barrier lyapunov functionsbased command filtered output feedback control for full-state constrained nonlinear systems," Automatica, vol. 105, pp. 71-79, 2019.

[7] J. Yu, P. Shi, and L. Zhao, "Finite-time command filtered backstepping control for a class of nonlinear systems," Automatica, vol. 92, pp. 173-180, 2018. 
[8] M. Singh, N. Sharma, A. Verma, and S. Sharma, "dynamic stochastic resonance based diffusion-weighted magnetic resonance image enhancement using multi-objective particle swarm optimization," Journal of Medical and Biological Engineering, vol. 36, no. 6, Article ID 891900, 2016.

[9] G. Maragatham and S. M. M. Roomi, "PSO-based stochastic resonance for automatic contrast enhancement of images," Signal, Image and Video Processing, vol. 10, no. 1, pp. 207-214, 2016.

[10] Y. Leng, E. Zhao, P. Shi, and Y. Zhang, "Image processing of two-dimensional stochastic resonance parameter adjustment," Journal of Tianjin University, vol. 10, pp. 907-913, 2011.

[11] T. Xu, H. Yu, J. Yu, and X. Meng, “Adaptive disturbance attenuation control of two tank liquid level system with uncertain parameters based on port-controlled Hamiltonian," IEEE Access, vol. 8, pp. 47384-47392, 2020.

[12] M. Singh, A. Verma, and N. Sharma, "Bat optimization based neuron model of stochastic resonance for the enhancement of MR images," Biocybernetics and Biomedical Engineering, vol. 37, no. 1, pp. 124-134, 2017.

[13] J. Liu, B. Hu, and Y. Wang, "Optimum adaptive array stochastic resonance in noisy grayscale image restoration," Physics Letters A, vol. 383, no. 13, pp. 1457-1465, 2019.

[14] J. Han, Q. Xu, J. Chen, L. Zhu, and Z. li, "Reconstruction of an underwater scattered image via incoherent modulation instability," Optics Letters, vol. 44, no. 3, pp. 695-698, 2019.

[15] G. Cui, J. Yu, and Q. G. Wang, "Finite-time adaptive fuzzy control for mimo nonlinear systems with input saturation via improved command-filtered backstepping," IEEE Transactions on Systems, Man, and Cybernetics: Systems, vol. 18, pp. 1-10, 2020.

[16] C. Fu, Q.-G. Wang, J. Yu, and C. Lin, "Neural network-based finite-time command filtering control for switched nonlinear systems with backlash-like hysteresis," IEEE Transactions on Neural Networks and Learning Systems, vol. 20, pp. 1-6, 2020.

[17] J. F. Lindner, B. K. Meadows, W. L. Ditto, M. E. Inchiosa, and A. R. Bulsara, "Array enhanced stochastic resonance and spatiotemporal synchronization," Physical Review Letters, vol. 75 , no. 1, pp. 3-6, 1995.

[18] N. Wang, B. Zheng, H. Zheng, and Z. Yu, "Feeble object detection of underwater images through LSR with delay loop," Optics Express, vol. 25, no. 19, pp. 22490-22498, 2017.

[19] F. Duan, F. Chapeau-Blondeau, and D. Abbott, "Input-output gain of collective response in an uncoupled parallel array of saturating dynamical subsystems," Physica A: Statistical Mechanics and Its Applications, vol. 388, no. 8, pp. 1345-1351, 2009.

[20] Z. Wang, Z. Qiao, L. Zhou, and L. Zhang, "Array-enhanced logical stochastic resonance subject to colored noise," Chinese Journal of Physics, vol. 55, no. 2, pp. 252-259, 2017.

[21] R. Srivastava, M. Dueñas-Díez, and J. Pérez-Mercader, "Feed rate noise modulates autocatalysis and shapes the oscillations of the belousov-zhabotinsky reaction in a continuous stirred tank reactor," Reaction Chemistry \& Engineering, vol. 3, no. 2, pp. 216-226, 2018.

[22] Y. Yao and J. Ma, "Weak periodic signal detection by sineWiener-noise-induced resonance in the fitzhugh-nagumo neuron," Cognitive Neurodynamics, vol. 12, no. 3, pp. 343-349, 2018.

[23] X. Shi and Z. Wang, "stability analysis of fraction-order hopfield neuron network and noise-induced coherence resonance," Mathematical Problems in Engineering, vol. 2020, Article ID 3520972, 58 pages, 2020.
[24] C. Nicolis and G. Nicolis, "Coupling-enhanced stochastic resonance," Physical Review E, vol. 96, no. 4, Article ID 042214, 2017.

[25] Y. Ergin, B. Veli, P. Matjaz, and O. Mahmut, "Enhancement of pacemaker induced stochastic resonance by an autapse in a scale-free neuronal network," Science China, vol. 59, no. 3, pp. 364-370, 2016.

[26] A. L. Hodgkin and A. F. Huxley, "A quantitative description of membrane current and its application to conduction and excitation in nerve," The Journal of Physiology, vol. 117, no. 4, pp. 500-544, 1952.

[27] R. Fitzhugh, "Impulses and physiological states in theoretical models of nerve membrane," Biophysical Journal, vol. 1, no. 6, pp. 445-466, 1961.

[28] T. Alarcón, A. Pérez-Madrid, and J. M. Rubí, "Stochastic resonance in nonpotential systems," Physical Review E, vol. 57, no. 5, pp. 4979-4985, 1998.

[29] J. J. Collins, C. C. Chow, and T. T. Imhoff, "Aperiodic stochastic resonance in excitable systems," Physical Review E, vol. 52, no. 4, pp. R3321-R3324, 1995.

[30] D. Guo, M. Perc, Y. Zhang, P. Xu, and D. Yao, "Frequencydifference-dependent stochastic resonance in neural systems," Physical Review E, vol. 96, no. 2, Article ID 022415, 2017.

[31] Y. Yao, L. Yang, C. Wang, Q. Liu, and R. Gui, "Subthreshold periodic signal detection by bounded noise-induced resonance in the fitzhugh-nagumo neuron," Complexity, vol. 2018, Article ID 5632650, 15 pages, 2018.

[32] H. Li, X. Sun, and J. Xiao, "Stochastic multiresonance in coupled excitable FHN neurons," Chaos: An Interdisciplinary Journal of Nonlinear Science, vol. 28, no. 4, 9 pages, Article ID 043113, 2018.

[33] J. Yu, P. Shi, C. Lin, and H. Yu, "Adaptive neural command filtering control for nonlinear MIMO systems with saturation input and unknown control direction," IEEE Transactions on Cybernetics, vol. 50, no. 6, pp. 2536-2545, 2020.

[34] C. W. Gardiner, Handbook of Stochastic Methods, pp. 80-115, Springer-Verlag, Berlin, Germany, 1985.

[35] Y. Yang, Z. Jiang, B. Xu, and D. W. Repperger, "an investigation of two-dimensional parameter-induced stochastic resonance and applications in nonlinear image processing," Journal of Physics A: Mathematical and Theoretical, vol. 42, no. 14, Article ID 145207, 2009. 